\title{
Joint Demographic Features Extraction for Gender, Age and Race Classification based on CNN
}

\author{
Zaheer Abbas ${ }^{1}$, Sajid Ali ${ }^{2}$, Muhammad Ashad Baloch ${ }^{3}$, Hamida Ilyas ${ }^{4}$, Moneeb Ahmad ${ }^{5}$ \\ Mubasher H. Malik ${ }^{6}$, Noreen Javaid ${ }^{7}$, Tanvir Fatima Naik Bukht ${ }^{8}$ \\ Department of Computer Science, Institute of Southern Punjab Multan, Pakistan 1, 4, 5, 6, 7, 8 \\ Department of Computer Science, University of Education Lahore, Pakistan ${ }^{2}$ \\ Department of Computer Science, NCBA\&E Multan, Pakistan ${ }^{3}$
}

\begin{abstract}
Automatic verification and identification of face from facial image to obtain good accuracy with huge dataset of training and testing to using face attributes from images is still challengeable. Hence proposing efficient and accurate facial image identification and classification based of facial attributes is important task. The prediction from human face image is much complex. The proposed research work for automatic gender, age and race classification is based on facial features and Convolutional Neural Network (CNN). The proposed study uses the physical appearance of human face to predict age, gender and race. The proposed methodology consists of three sub systems, Gender, Ageing and Race. Therefore different feature are extracted for every sub system. These features are extracted by using Primary, Secondary features, Face Angle, Wrinkle Analysis, LBP and WLD. The accuracy of classification is based on these features. CNN used to classify by using these features. The proposed study has been evaluated and tested on large database MORPH II and UTKF. The performance of proposed system is compared with state of art techniques.
\end{abstract}

Keywords-Appearance features; age; gender; wrinkle analysis; face angle; classification; race; $\mathrm{LBP}$

\section{INTRODUCTION}

The face of human holds significant amount of qualities and data about the human identification, for example, appearance, race, gender classification, and age. Humans can identify and examine these data effectively, for example, most of human can perceive human attributes like gender classification [1], and they can judge if the human is male or female by just observing face. They can estimate the age of the individual and state whether this individual is a kid or a grown-up. Without a doubt, most of programmed facial characteristic arrangement frameworks depend on machine vision and machine acknowledgment. Computer vision incorporates strategies and procedures for comprehension, breaking down, and removing data from pictures. In [2] the plan of a machine acknowledgment framework includes three principle parts, which are pre-handling, highlights extraction, and order. Age predicting gender classification and race forecast frameworks has been developing quickly as of late due its significant modules and valuable uses for some machine vision applications in seeing a particular portion of individuals. Estimation of gender and age forecast frameworks are presently being utilized by lodgings, air terminals, transport stations, club, government stations, colleges, clinics, films, and so forth to build the degree of security and tackling any potential threats or lacks. In the security apps, age and gender forecast methods are utilized likewise in social insurance frameworks, data recovery, scholarly examinations and investigates, and (ECRM) frameworks, customers are conveyed to various gender classification and age gatherings like youngsters, adolescents, old citizens and senior old citizens not withstanding decide if they male or female. Moreover, assembling some client's day by day life data like exercises, propensities, customs, needs and so forth may assist the enterprises with classifying items and administrations relying upon their gender classification or age gatherings that lead to expand their earnings and procure more cash [3]. In [4] For instance, clothing stores may offer suitable styles for guys or females as per their age gatherings; cafés required to know the mass prominent suppers for each age or gender classification gathering, multiple organizations need to make explicit publicizing to explicit spectators relying upon their gender, age and race estimation structures [4].

There are totally different algorithmic program and techniques are already for age, gender, and race classification severally or combination of two techniques. There's no multitasked design for age, gender and race classification conjointly. The problem statement is taken from [22]. The planned work is organized for race, age and gender estimation conjointly, during this study multilayer design developed for age, gender and race classification supported by Convolutional Neural Network (CNN). All structured are learned and radiocontrolled by auxiliary demographic data, since alternative demographic data (i.e., gender and race) is helping for age estimation. Every cascaded structure is embodied in an exceedingly main network and several other sub networks. The applied frameworks gender trained by gender data, then two sub networks are trained by the male and female samples, severally. We have a tendency to use the options extracted from the physically structure with mathematician method regression that may enhance the performance for age estimation, gender prediction and race classification. The Experimental results on the MORPH II and UTKF datasets have gained superior performances compared to the progressive ways with state of art techniques. This paper organized as follow, Section 2 described related work. The Methodology is delineated in the Section 3. Experimental result discussed in Section 4. The conclusion and future work is presented in Section 5. 


\section{RELATED WORK}

Facial image recognizable research has consisted 30 decades as stated by previous work. However face traits, for example, age, gender orientation and ethnicity recognizable proof from facial pictures has effectively developing in late $1996 \mathrm{~s}$ as per the previous. Research on facial quality acknowledgment, for example, age, gender, ethnicity and facial feelings have been begun almost 10 years back. In this way there are just a couple of research arrangements presented for facial property grouping from human face image. It is fundamental to present new techniques with higher correctness for face characteristic grouping from facial images. As indicated by the writing, the majority of the exploration arrangements depend on characterizing the pictures into one of the traits yet not for a mix of properties. The absolute first association did with age grouping from facial pictures in 1999 by Y. H. Kwon et, al [5]. Wen-Bing Horng et al. [6] built up a calculation to distinguish age from gray level pictures by utilizing an Artificial Neural Network (ANN) classifier. Proposed strategy can characterize pictures into one of the four classifications from children, young, old, moderately aged, and older aged. K B Raja et al [7] proposed an estimation period of life and gender orientation classifier utilizing ANN framework and back class likelihood. The calculation comprises of three primary stages, preprocessing, include extraction and characterization. Feng GAO and Haizhou Ai [8] built up their own calculation to confront the test of age order utilizing buyer pictures in different state. Gabor highlight is separated for face image portrayal and a fluffy rendition of Linear Discriminate Analysis is utilized for arrangement. They [9] exhibited a novel strategy for face gender characterization by utilizing totally skewed information, for example, made an interpretation of or turned information into the preparation set and got a momentous exactness. Proposed approach utilized two classifiers for the examination. One depends on feeble highlights; for example, LBP histograms depend on SIFT key focuses. At long last the proposed framework has accomplished a high precision of $92.5 \%$. The neighborhood qualities are usually known to all the more likely arrange an individual into age bunches as they insert specific attributes that separate distinctive age gatherings. Instead of the neighborhood highlights, they contended that the worldwide partners are better for evaluating increasingly exact age data and contain the age characteristics, however further individual-related qualities as personality, feeling and ethnic foundation. Half breed highlights which are created through joining nearby and worldwide highlights are found to offer unrivaled execution for different face related applications. This is a direct result of the wastefulness found in each sort of highlight can be remunerated prompting the end that cross breed highlights are alluring for precise age estimation. One of the prior chips away at sexual orientation acknowledgment utilizing CNNs was exhibited in paper [10]. The arrangement comprised of a face location and sexual orientation acknowledgment module - both utilizing neural systems. The engineering included three layers (two covered up and yield). Info pictures had $32 \times 32$ pixels goals. The announced precision on the FERET dataset was $97.2 \%$. A large portion of the business related to the DCNNs showed up in 2015 and later. In the article two methodologies were thought about: "great" and DCNN based. In the first case the accompanying highlights were considered: HOG, LBP and SURF. As relapse the CCA (Canonical Correlation Analysis) was connected. In the second, a wide range of variations of system designs were inspected (the Caffe library was utilized). The arrangement comprised of a face discovery and sexual orientation acknowledgment module - both utilizing neural systems. The engineering included three layers (two covered up and yield). Information pictures had a $32 \times 32$ pixels goals. The revealed exactness on the FERET dataset was $97.2 \%$. The vast majority of the business related to the DCNNs showed up in 2015 and later. In the article [10] two methodologies were looked at: "great" and DCNN based. In the first case the accompanying highlights were considered: HOG, LBP and SURF. As relapse the CCA (Canonical Correlation Analysis) was connected. In the second, various variations of system designs were inspected (the Caffe library was utilized). The best outcome was gotten for two Convolutional and one completely associated layer. Info pictures had $50 \times 50$ pixels size. The creators noticed significantly lopsidedness between the time required for learning and real activity in the two cases. At last, for the MORPH database, the "work of art" arrangement got 4.25 and DCNN 3.88 mean outright blunder (MAE) esteem. In the work [11] a DCNN for age and sexual orientation estimation was proposed. The system had three Convolutional and two completely associated layers. Information pictures of size $256 \times 256$ were edited to $227 \times$ 227. The creators did not utilize a pre-prepared system model opposed to numerous different methodologies. There are totally different algorithmic program and techniques are already for age, gender, and race classification severally or combination of 2 techniques. There's no multitasked design for age, gender and race classification conjointly. The planned work is organized for age, gender and race estimation conjointly, during this study multilayer design developed for age, gender and race classification supported Convolutional Neural Network (CNN). All structured are learned and radiocontrolled by auxiliary demographic data, since alternative demographic data (i.e., gender and race) is helping for age estimation. Every cascaded structure is embodied in an exceedingly main network and several other sub networks. as an example, one in every of the applied frameworks may be a gender trained by gender data, then 2 sub networks are trained by the male and female samples, severally. We have a tendency to use the options extracted from the physically structure with mathematician method regression that may enhance the performance for age estimation, gender prediction and race classification. The Experimental results on the MORPH II and UTKF datasets have gained superior performances compared to existing techniques.

\section{Proposed Methodology}

The main purpose of proposed system is estimated jointly gender, age and race on the basis of specific human facial features. The human extraction of facial features is important phase in the proposed algorithm. Fig. 1 demonstrates the proposed system architecture flow that clearly shows that the most important steps in proposed system (preprocessing, Feature extraction and classification). 
Primary landmarks getting is the more significant stage in the examination. Primary landmarks in the face image are nose, eyes, eyebrow and mouth. They present a particular district where every face component falls in a picture and in this manner. The quest landmark for a facial element could be limited. As per this perception, face region can be separating into a few pieces and begin to find each element point utilizing a prepared classifier for each component. Each element is found utilizing prepared classifiers for every element. Face districts are first sent to the comparing identifier to identify the separate component regions. At that point subsequent stage is to discover the closest enormous enough district including the found component territory.

\section{A. Gender Classification}

A particular number of parameters are needed to proceed with gender prediction. Fig. 2 demonstrates the used features of gender classification.

The description of the parameters is given below:

$\hat{\mathrm{E}}=\frac{X_{1}+X_{2}}{2}$

Here $\hat{E}$ is height of eye.

$\rho=\frac{Y_{1}+Y_{2}}{2}$

Here $\rho$ Distance between Eye and Eye brow.

$\Gamma=\frac{b(d+f)}{2}$

Here $\Gamma$ height of Nose.

$\omega=\mathrm{h}-\mathrm{b}$

Here $\omega$ space between Nose and Lip.

Eyebrow width $=\mathrm{Q}$

$\tau=\mathrm{e}-\mathrm{c}$

Here $\tau$ distance between Eyes.

$\lambda=\frac{h-(f+d)}{2}$

Here $\lambda$ Eye to upper Lip distance.

$R_{1}=\frac{\varsigma}{\Gamma}$

Here $\varsigma$ distance between Eyes and $\Gamma$ height of Nose.

$R_{2}=\frac{\varsigma}{\lambda}$

Here $\varsigma$ distance between Eyes and $\lambda$ Eye to upper Lip distance.

\section{B. Age Estimation}

The features of appearance face have consisted of two features, shape and facial skin texture features. During the changes in the age from childhood to adult the shape features also changed considerably. The shape features are very helpful for estimating the age of adult instead of babies. In other hand facial skin texture feature is more efficient to differentiate between adult and senior adult. Thus texture and shape features are provided useful information for estimating age.
Therefore facial texture and shape features are used to develop age estimation phase. In the proposed age estimation phase face angle and geometric ratios are used as shape landmarks and (LGBPH) [12] of skin features is used as texture features.

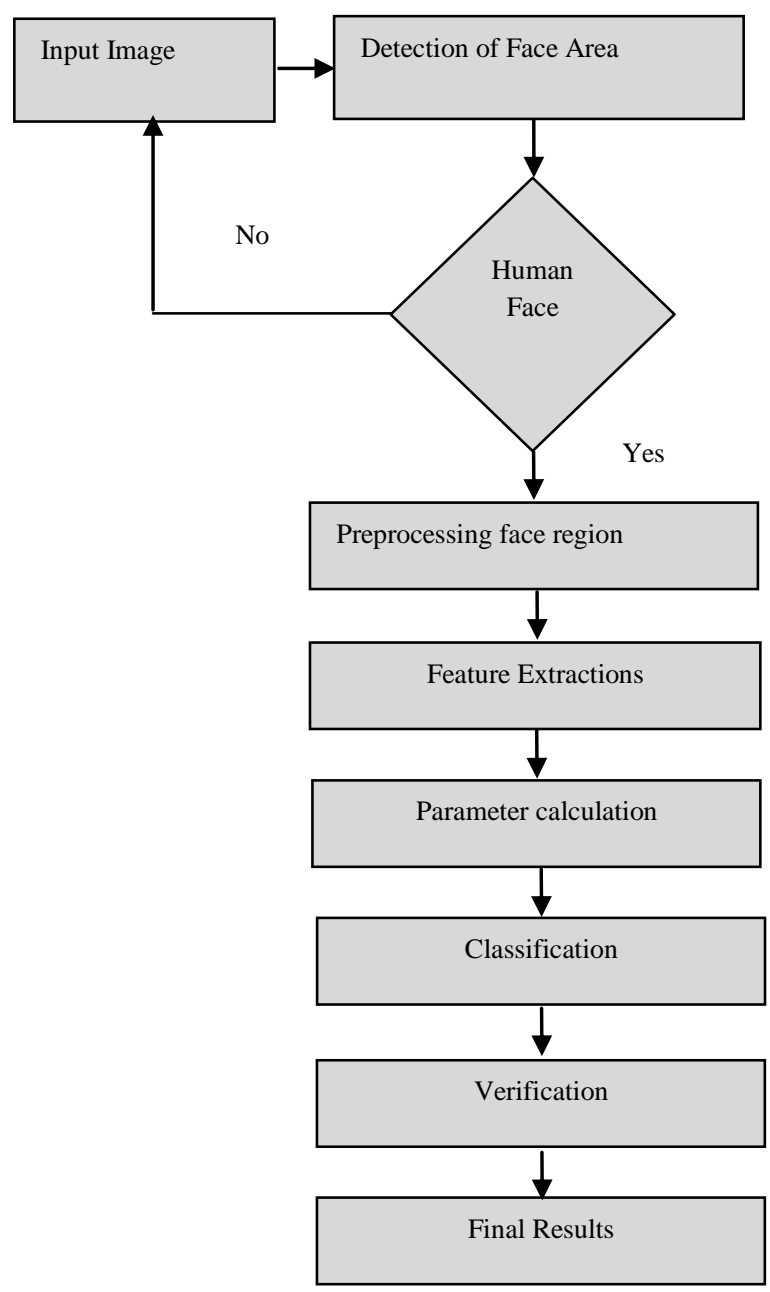

Fig. 1. Demonstrate Flow of Proposed System.

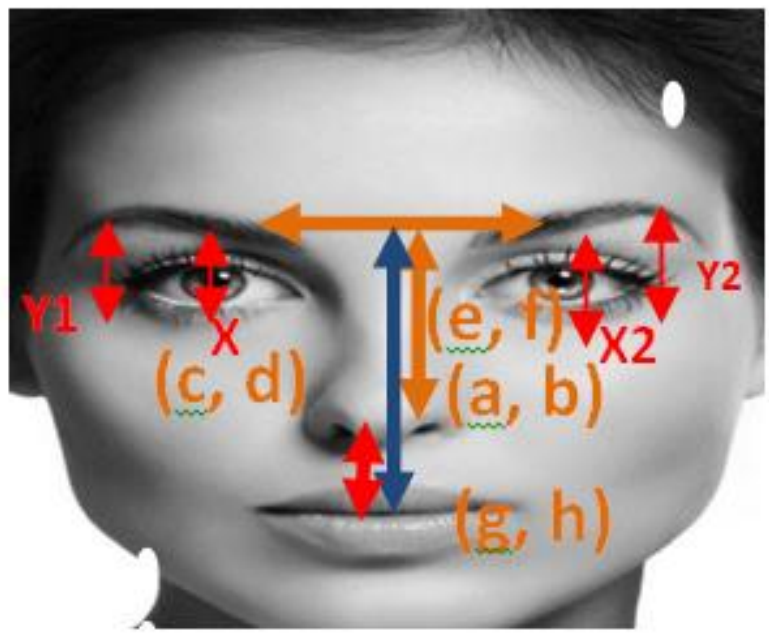

Fig. 2. Gender Classification Parameter. 
The general equation of calculating ratios is follow:

$R_{i}=\frac{D_{h i}}{D_{v i}}$

$\mathrm{R}_{\mathrm{i}}$ is demonstrate the ratio which are calculate by using vertical and horizontal distance between different face angles $\mathrm{D}_{\mathrm{hi}}$ horizontal distances and $\mathrm{D}_{\mathrm{vi}}$ vertical distance.

$R_{1}=\frac{D_{\text {heni }}}{D_{\text {veei }}}$

$D_{\text {heni }}$ Span middle of eyes and nose tip $D_{\text {veei }}$ span middle of eyes.

$R_{2}=\frac{D_{\text {hemi }}}{D_{\text {veei }}}$

$D_{\text {hemi }}$ Span middle of eyes and centre of mouth $D_{\text {veei }}$ span middle of eyes.

$R_{3}=\frac{D_{\text {heci }}}{D_{\text {veei }}}$

$D_{\text {heci }}$ Span middle of eyes and chin tip $D_{\text {veei }}$ span middle of eyes.

$R_{4}=\frac{D_{\text {heni }}}{D_{\text {vemi }}}$

$D_{\text {heni }}$ Span middle of eyes and nose tip $D_{\text {vemi }}$ span middle of eyes and centre of mouth.

$R_{5}=\frac{D_{h e n i}}{D_{\text {veci }}}$

$D_{\text {heni }}$ Span middle of eyes and nose tip $D_{\text {veci }}$ span middle of eyes and chin tip.

$R_{6}=\frac{D_{\text {heni }}}{D_{\text {veci }}}$

$D_{\text {heni }}$ Span middle of eyes and centre of mouth $D_{\text {veci }}$ span middle of eyes and Chin tip.

1) Face angle: The angle of face image is extracted as demonstrate in Fig. 3 is measured by applying the law of cosines, which used to extract as another shape landmark in the presented age estimation architecture.

2) Facial skin textural features: The characteristics of facial skin extracted from LGBPH described by Zhang et al. (2005) have been used successfully for face recognition and their effectiveness has been proven in comparison to conventional LBP features or Gabor features.

3) Wrinkle analysis: The wrinkle analysis comprises of wrinkle highlight extraction utilizing LGBPH and wrinkle thickness includes extraction. Wrinkle includes extraction utilizing LGBPH is completed with slight adjustments in the method talked about for face skin textural highlight extraction utilizing LGBPH. The reaction of Gabor channel is a most extreme toward wrinkles.

$W_{d}=\frac{W_{p}}{T_{p}}$

The wrinkle density is represented by $\mathrm{W}_{\mathrm{d}}$, the count of wrinkle pixels and the count of total pixels in the region of interest is represented by $\mathrm{W}_{\mathrm{p}}$ and $\mathrm{T}_{\mathrm{p}}$, respectively.

\section{Race Classification}

The proposed race identification methods are used to extract standardized information from face pictures: LPB and WLD [13]. The CNN classifier is utilized for distinguishing the race. We have experimented with numerous distance measures.

1) Local binary pattern: LBP has been the for the most part used descriptor and best performing surface descriptors in present years. This administrator names the pixels of a picture by thresholding the $3 \times 3$ neighborhood of each pixel with the inside worth and thinking about the outcome as a double number.

The central value from Fig. 4 is calculated by the binary values which obtained by writing clock wise binary number from $3 * 3$ matrix.

$\mathrm{CW}=11000110=198$

The LBP is computed using the following equation:

$$
L B P_{P . R}=\sum_{i-1}^{P-1} 2^{i} \times S\left(P_{i}-P_{c} \quad\right)
$$

Where $\mathrm{P}_{\mathrm{c}}$ is the center pixel and the thresholding operation is

$\mathrm{S}(\mathrm{Pi}-\mathrm{Pc})=\left\{\begin{array}{l}1, p_{i}-p_{c} \geq 0 \\ 0, P_{i}-p_{c}<0\end{array}\right.$

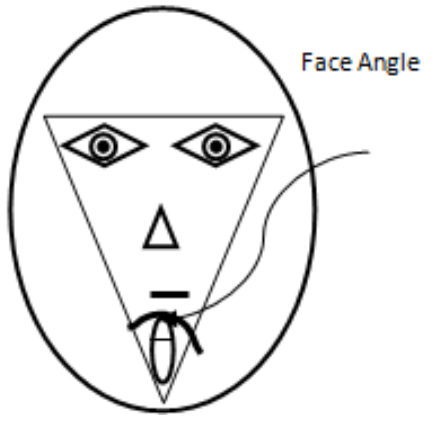

Fig. 3. Face Angle.

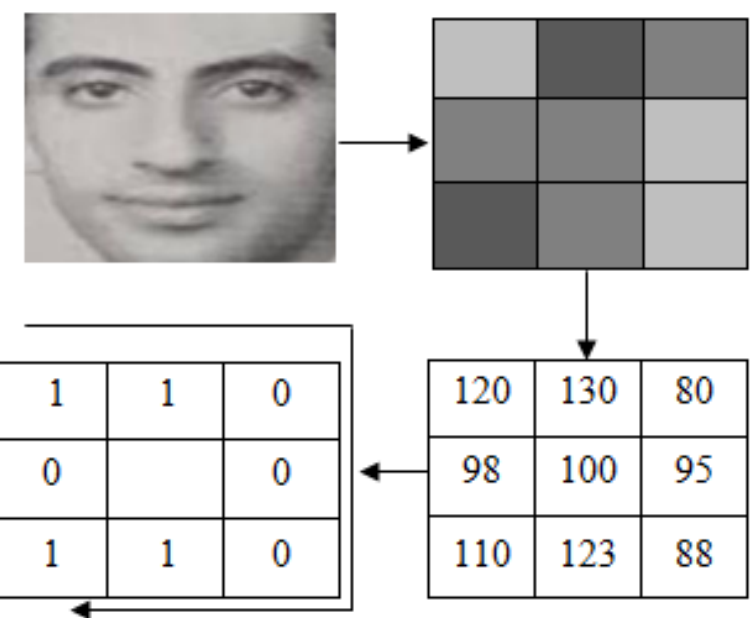

Threshold

Fig. 4. Shows the LBP Operator. 
2) Weber Law Descriptor: WLD can be a solid and ground-breaking nearby handle as of late created. It is blend of two sub parts: differential excitation and direction. The WLD was dazzled by a mental law known as the Weber Law [13]. The proposed law shows that the difference in information, (for example, sound, lighting) that might be felt might be a consistent proportion of the first info. Since the progressions are not as much as this association with a fixed worth, the animal would remember it as a foundation signal rather than a genuine sign and concentrate the sides of the picture accurately even within the sight of noteworthy commotion and is solid against light change.

\section{Convolutional Neural Network}

Classification takes place in three main steps. Gender classification first identifies the gender of source image. Fig. 5 demonstrates the structure of CNN.

Then source image forward to the age classifier to identify the relative age order and forward to the race classifier to classify the race. Gender classification is basically using the facial features on features of face, age range based on wrinkle analysis, face angle and the classification of the race, which is done LBP and WLD. Identification process completed by utilizing calculated parameters of Convolutional Neural Networks. Convolutional Neural networks are trained using data taken from nearly 2,400 images of two genders, different age groups and four races, which are front and near front images. Fig. 5 shows the structure of the neural network used for age classification. The parameters are sent to the $\mathrm{CNN}$ to classify the images per gender, age and race. CNN used for gender prediction having 5 input and 2 output nodes 0 and 1 resembling the 2 gender teams, male $(0-0.5)$ and feminine $(0.5$ - 1.0). Output worth from the Convolutional Neural Network is employed to classify the image into one amongst the gender teams. The neural network wont to classify the given input image in to a corresponding age bracket has eight input nodes and also the output layer contains four nodes particularly zero, $1,2,3,4,5,6$ and 7 to represent the age teams $0-5,6-11,12-17,18-30,31-60,61-74$ and 75-95 respectively. The somatic cell with the best response is taken into account because the output of the age orders CNN projection technique. Thus manual classification takes place for the incorrectly detected pictures.

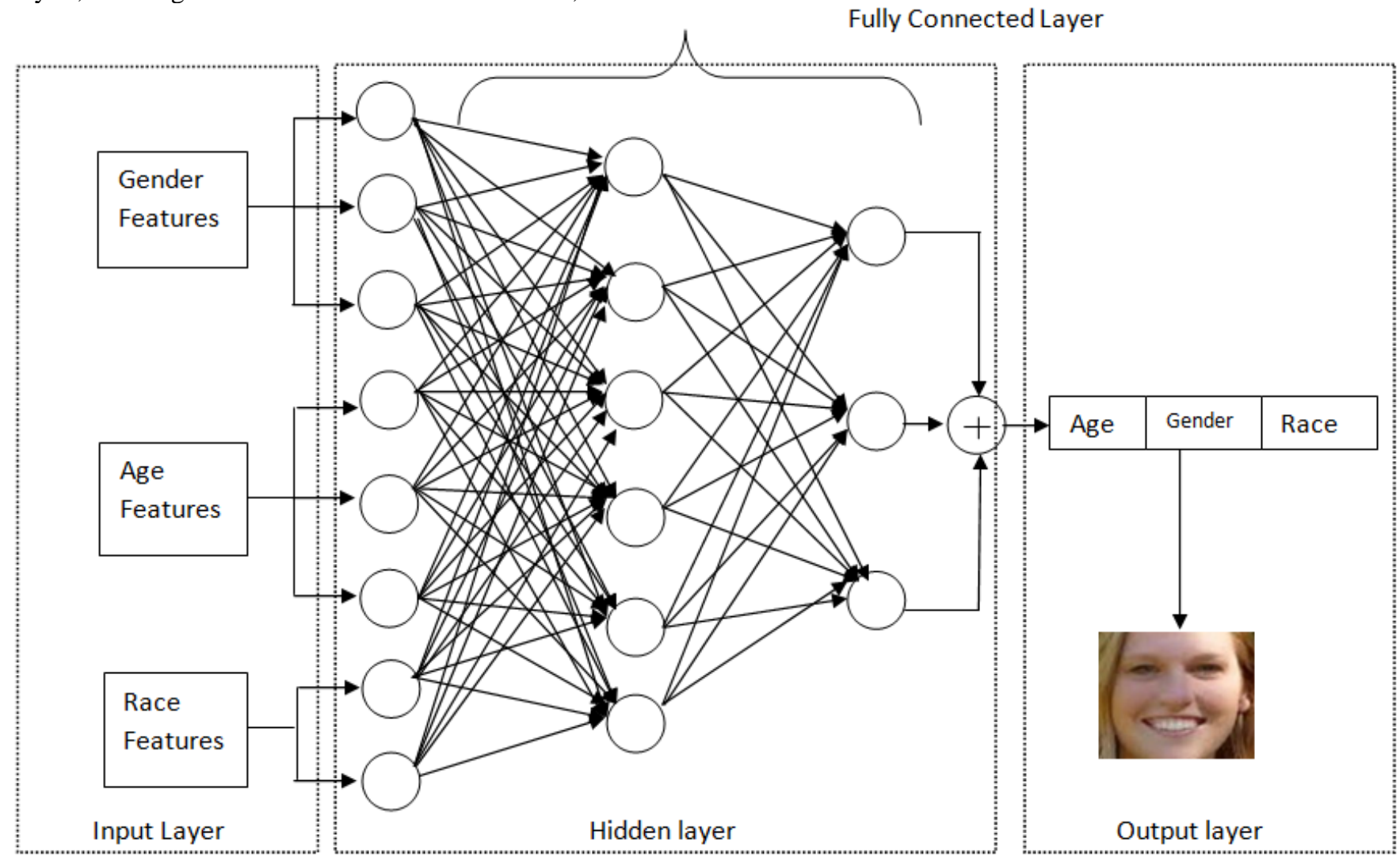

Fig. 5. Neural Network Framework for Age, Gender and Race Classification. 


\section{RESULTS AND EXPERIMENTS}

The proposed work is organized for gender, age and race estimation conjointly, during this study multilayer design developed for age, gender and race classification supported Convolutional Neural Network (CNN). All structured are learned and radio-controlled by auxiliary demographic data, since alternative demographic data (i.e., gender and race) is helping for age estimation. Every cascaded structure is embodied in an exceedingly main network and several other sub networks. as an example, one in every of the applied frameworks may be a gender trained by gender data, then two sub networks are trained by the female and male samples, severally. We have a tendency to use the feature extraction from the physically structure with mathematician method regression that may enhance the performance for age estimation, gender prediction and race classification. The Experimental results on the UTKF and MORPH II datasets have gained superior performances comparison in progressive ways with state of art techniques. The experiments compiled on Lenovo i3, 6 GB RAM, windows 10 operating system, Matlab 2019. The proposed system Gender, Age and Race classification trained and testing on MORPH II and UTKF faces database. More than $2400+$ images are trained and number of test applies on trained dataset. The propose framework are consisted on three phases which are briefly described in chapter 3 . The first phase is performing preprocessing on input image and pass to second phase. It is most important phase of proposed framework, it calculated feature of input image for age, gender and race. After successfully extracting features (the feature extracting techniques are briefly described in Section 3), the results will pass to third phase, in this the phase classification were done by using CNN. We trained more than 2400 images and more than 1200 images are tested for experiments. The result are extracted from testing images are follow. Gender prediction performance accuracy is extremely dependent on specific landmarks that extract from input face. Landmarks just like height of eye (D1), span of eye brow corner and eye corner (D2), nose height (D3), span of nose and lip (D4) and breadth of eye brow (D5). Set of results taken for the five parameters from the take a look at image is shown within the Table I. These parameters in brief delineate in Section 3.

Tables II, III and V show the confusion matrix of gender, age and race, respectively.

The performance of projected framework is described by exploitation Table III referred to as confusion matrix. The first column of Table III shows actual seven age classes of the input face photos. Initial row of Table III shows foretold age classes of the input take a glance at face photos by the projected age organization. The count inside the diagonal cell of Table III is incremented, if the anticipated class is same as actual class for the input take a glance at face image. Therefore, the diagonal entries of Table III shows the count of accurately classified input take a glance at face photos for corresponding age classes. Non-diagonal entries of Table III show the count of misclassified input take a glance at face photos. Total of count of accurately classified and misclassified entries across the rows of Table III offers total type of face photos inside the express people. The illustration confusion matrix in graphs clearly shows the accuracy oldtime detection. Some inaccurate results jointly show in confusion matrix and graphs that indicate the wrong detection by projected style. In this paper, we use the MAE for accuracy calculation. For the MAE calculation, it computes he MAE between the true age and the predicted age in the testing set. Formally, MAE is calculated as:

$M A E=\frac{1}{N} \sum_{m=1}^{N}\left|l_{m}-l_{m}^{\prime}\right|$

Where $l_{\mathrm{m}}$ and $l_{\mathrm{m}}^{\prime}$ denotes the ground truth age and predicted age of the $\mathrm{m}_{\mathrm{th}}$ image, respectively, and $\mathrm{N}$ is the number of testing images. If the value of MAE is lower, the performance is better. Table IV demonstrates the age results and Fig. 6 shows graphical representation on MORPH II database.

TABLE. I. PARAMETERS VALUES FOR GENDER CLASSIFICATION

\begin{tabular}{|l|l|l|l|l|l|}
\hline Image & D1 & D2 & D3 & D4 & D5 \\
\hline A01 & 0.20 & 0.30 & 0.55 & 0.23 & 0.08 \\
\hline A02 & 0.16 & 0.14 & 0.55 & 0.36 & 0.06 \\
\hline N01 & 0.16 & 0.20 & 0.64 & 0.32 & 0.11 \\
\hline N02 & 0.14 & 0.13 & 0.63 & 0.37 & 0.13 \\
\hline A03 & 0.16 & 0.17 & 0.53 & 0.27 & 0.06 \\
\hline A04 & 0.16 & 0.21 & 0.64 & 0.24 & 0.6 \\
\hline N03 & 0.17 & 0.14 & 0.51 & 0.35 & 0.10 \\
\hline N04 & 0.16 & 0.28 & 0.67 & 0.31 & 0.8 \\
\hline A05 & 0.16 & 0.25 & 0.65 & 0.25 & 0.08 \\
\hline
\end{tabular}

TABLE. II. SHOWS CONFUSION MATRIX OF GENDER ClASSIFICATION

\begin{tabular}{|l|l|l|}
\hline & Male & Female \\
\hline AG1 & 98 & 2 \\
\hline AG2 & 1 & 99 \\
\hline AG3 & 99 & 1 \\
\hline AG4 & 2 & 98 \\
\hline
\end{tabular}

TABLE. III. SHOWs CONFUSION MATRIX OF AgE CLASSIFICATION

\begin{tabular}{|l|l|l|l|l|l|l|l|}
\hline & S1 & S2 & S3 & S4 & S5 & S6 & S7 \\
\hline AGS1 & 116 & 3 & 1 & 0 & 0 & 0 & 0 \\
\hline AGS2 & 2 & 114 & 2 & 2 & 0 & 0 & 0 \\
\hline AGS3 & 1 & 3 & 110 & 3 & 3 & 0 & 0 \\
\hline AGS4 & 0 & 0 & 1 & 87 & 2 & 0 & 0 \\
\hline AGS5 & 0 & 0 & 1 & 1 & 87 & 1 & 0 \\
\hline AGS6 & 0 & 0 & 0 & 1 & 2 & 85 & 2 \\
\hline AGS7 & 0 & 0 & 0 & 0 & 2 & 2 & 86 \\
\hline
\end{tabular}

TABLE. IV. EXPERIMENTAL RESUltS ON MORPH II DATABASE

\begin{tabular}{|l|l|}
\hline Method & MAE \\
\hline AGES [14] & 8.83 \\
\hline CA-SVR [15] & 5.88 \\
\hline OHRank [16] & 5.69 \\
\hline DLA [17] & 4.77 \\
\hline VGG+SVR [18] & 3.45 \\
\hline DEX [19] & 3.33 \\
\hline OURS & 3.29 \\
\hline
\end{tabular}




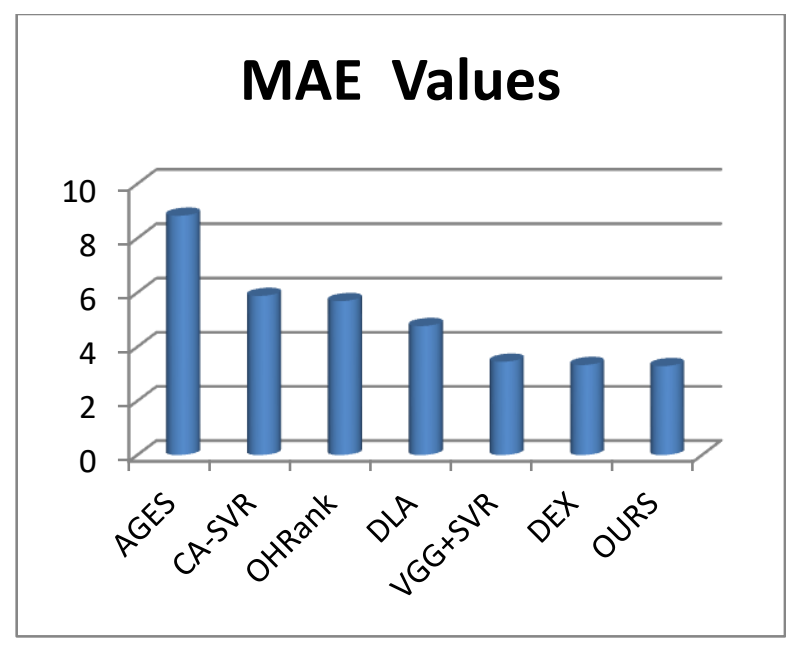

Fig. 6. Comparison with State of the Art Existing Technique.

Fig. 7 demonstrates the race accuracy on different races (Mongolian, Indian, Black, and White).

In Table VI shows the result comparisons with state of art techniques.

Fig. 8, 9 and 10 show the overall accuracy of proposed accuracy with other state of art techniques gender age and race respectively. In the proposed study we have been create a framework to classify age, gender and race jointly. The proposed work based on facial human feature. The feature extracted by using different appearance features. The experiments clearly show in this chapter the proposed work better than existing techniques. The proposed performance compared with state of art techniques overall and also by sub modules. Every module and overall performance clearly demonstrated that the proposed system work comparatively better.

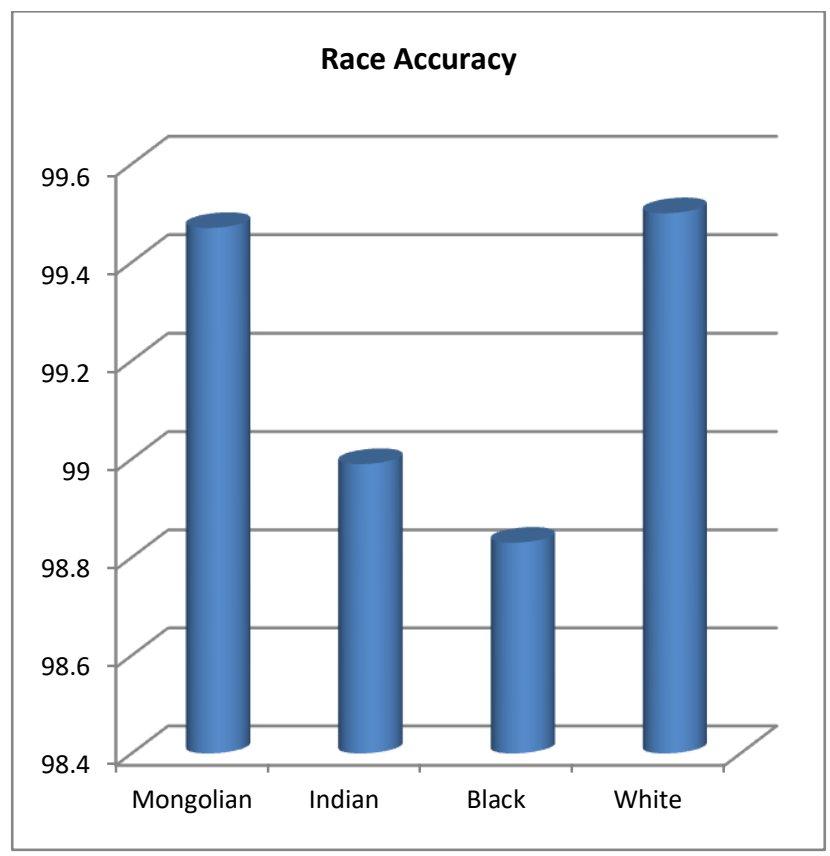

Fig. 7. Shows Accuracy of Race Classification.
TABLE. V. CONFUSION MATRIX OF RACE ACCURACY

\begin{tabular}{|l|l|l|l|l|}
\hline & Mongolian & Indian & White & Black \\
\hline AG1 & $99.47 \%$ & - & - & $0.53 \%$ \\
\hline AG2 & - & $98.99 \%$ & - & $1.01 \%$ \\
\hline AG3 & - & - & $98.83 \%$ & $1.17 \%$ \\
\hline AG4 & $0.25 \%$ & $0.25 \%$ & - & $99.50 \%$ \\
\hline
\end{tabular}

TABLE. VI. DEMONSTRATE THE COMPARISON OF PROPOSED METHOD WITH STATE OF ART TECHNIQUES

\begin{tabular}{|c|c|c|c|c|c|}
\hline $\mathrm{M}$ & $\mathrm{TS}$ & GA & RA & $\mathrm{AM}$ & AM \\
\hline \multirow{2}{*}{ CCA [20] } & $S_{1}$ & $95.2 \%$ & $97.8 \%$ & 5.39 & \multirow{2}{*}{5.37} \\
\hline & $S_{2}$ & $95.2 \%$ & $97.8 \%$ & 5.35 & \\
\hline \multirow{2}{*}{$\begin{array}{l}\text { KCCA } \\
{[20]}\end{array}$} & $S_{1}$ & $98.5 \%$ & $98.9 \%$ & 4.00 & \multirow{2}{*}{3.98} \\
\hline & $S_{2}$ & $98.4 \%$ & $99.0 \%$ & 3.35 & \\
\hline \multirow{2}{*}{ PLS [21] } & $S_{1}$ & $97.3 \%$ & $98.7 \%$ & 4.58 & \multirow{2}{*}{4.56} \\
\hline & $S_{2}$ & $97.4 \%$ & $98.6 \%$ & 4.54 & \\
\hline \multirow{2}{*}{ KPLS [21] } & $S_{1}$ & $98.4 \%$ & $99.0 \%$ & 4.07 & \multirow{2}{*}{4.04} \\
\hline & $\mathrm{S}_{2}$ & $98.3 \%$ & $99.0 \%$ & 4.01 & \\
\hline \multirow{2}{*}{ OURS } & $S_{1}$ & $98.69 \%$ & $99.1 \%$ & 3.29 & \multirow{2}{*}{3.31} \\
\hline & $\mathrm{S}_{2}$ & $98.60 \%$ & $99.2 \%$ & 3.33 & \\
\hline
\end{tabular}

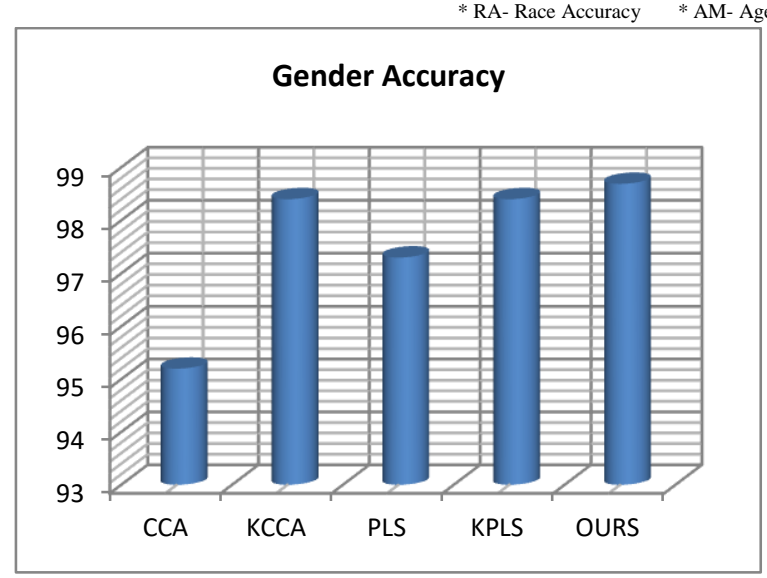

Fig. 8. Overall Gender Accuracy.

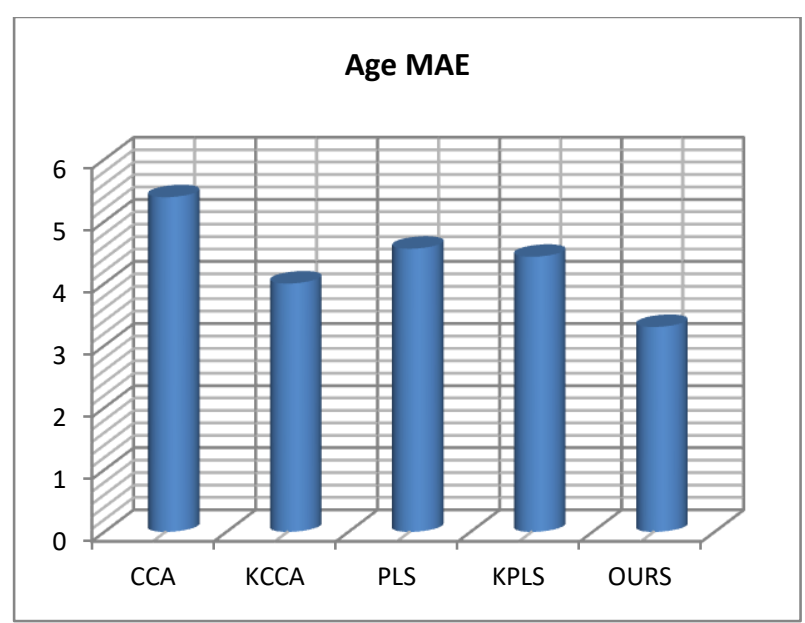

Fig. 9. Demonstrate the Overall MAE of Age. 


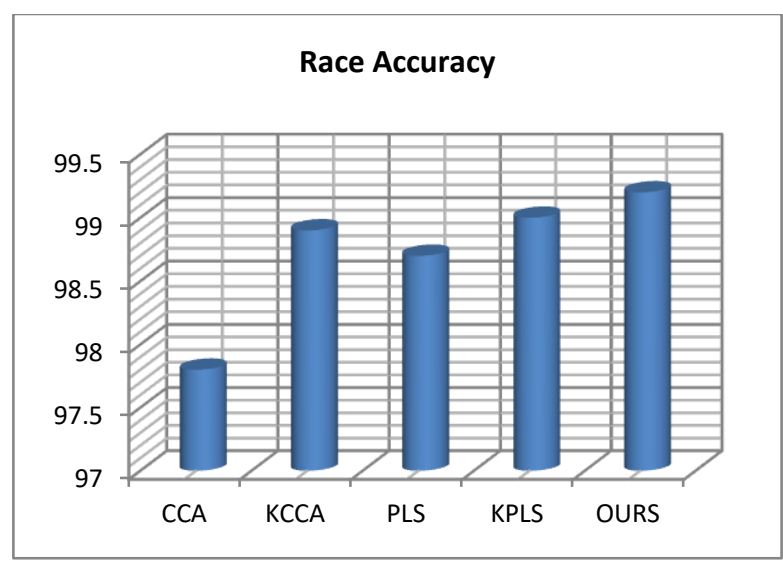

Fig. 10. Demonstrate the Overall Race Accuracy.

\section{CONCLUSION}

The proposed architecture based on Convolutional Neural Network, which is learned by Primary, Secondary, Wrinkle analysis, LBP and WLD. The proposed system classify the gender, age and race very efficiently. The overall accuracy of proposed work is $98 \%$. The sub module accuracy is $98.63 \%$ for gender classification, $99.2 \%$ for Race prediction and 3.31 MAE for age estimation. For the verification the efficiency of the proposed methods, we tested them on several databases. Consequently, we got nearly the same performance on two different databases (MORPH II and UTKF). The overall performance of proposed system is very efficient. The proposed system results compare with state of art methods. The comparison results show that the proposed system detect gender, age and race is more accurately than existing techniques. Generally, from the performance of proposed methods, we noticed that CNN classifiers more accurate than SVM, KNN and ANN. Our future work will focus to design such a framework that can detect age, race and gender on the basis of single appearance feature.

\section{REFERENCES}

[1] Nguyen, P., Tran, D., Huang, X., \& Ma, W. (2013, November). Age and gender classification using EEG paralinguistic features. In 2013 6th International IEEE/EMBS Conference on Neural Engineering (NER) (pp. 1295-1298). IEEE.

[2] Eidinger, E., Enbar, R., \& Hassner, T. (2014). Age and gender estimation of unfiltered faces. IEEE Transactions on Information Forensics and Security, 9(12), 2170-2179.

[3] Khan, S. A., Ahmad, M., Nazir, M., \& Riaz, N. (2014). A comparative analysis of gender classification techniques. Middle-East Journal of Scientific Research, 20(1), 1-13.

[4] Lin, F., Wu, Y., Zhuang, Y., Long, X., \& Xu, W. (2012). Human Gender Classification: A Review. International Journal of Biometrics.
[5] Kwon, Y. H., \& da Vitoria Lobo, N. (1999). Age classification from facial images. Computer vision and image understanding, 74(1), 1-21.

[6] Horng, W. B., Lee, C. P., \& Chen, C. W. (2001). Classification of age groups based on facial features. 淡江理工學刊, 4(3), 183-192.

[7] Ramesha, K. B. R. K., Raja, K. B., Venugopal, K. R., \& Patnaik, L. M. (2010). Feature extraction based face recognition, gender and age classification.

[8] Gao, F., \& Ai, H. (2009, June). Face age classification on consumer images with gabor feature and fuzzy lda method. In International Conference on Biometrics (pp. 132-141). Springer, Berlin, Heidelberg.

[9] Mayo, M., \& Zhang, E. (2008, November). Improving face gender classification by adding deliberately misaligned faces to the training data. In 2008 23rd International Conference Image and Vision Computing New Zealand (pp. 1-5). IEEE.

[10] Tivive, F. H. C., \& Bouzerdoum, A. (2006, July). A gender recognition system using shunting inhibitory convolutional neural networks. In The 2006 IEEE International Joint Conference on Neural Network Proceedings (pp. 5336-5341). IEEE.

[11] Levi, G., \& Hassner, T. (2015). Age and gender classification using convolutional neural networks. In Proceedings of the iEEE conference on computer vision and pattern recognition workshops (pp. 34-42).

[12] Hermosilla, G., Ruiz-del-Solar, J., Verschae, R., \& Correa, M. (2012). A comparative study of thermal face recognition methods in unconstrained environments. Pattern Recognition, 45(7), 2445-2459.

[13] Chen, J., Shan, S., He, C., Zhao, G., Pietikainen, M., Chen, X., \& Gao, W. (2009). WLD: A robust local image descriptor. IEEE transactions on pattern analysis and machine intelligence, 32(9), 1705-1720.

[14] Geng, X., Zhou, Z. H., \& Smith-Miles, K. (2007). Automatic age estimation based on facial aging patterns. IEEE Transactions on pattern analysis and machine intelligence, 29(12), 2234-2240.

[15] Chen, K., Gong, S., Xiang, T., \& Change Loy, C. (2013). Cumulative attribute space for age and crowd density estimation. In Proceedings of the IEEE conference on computer vision and pattern recognition (pp. 2467-2474).

[16] Chang, K. Y., Chen, C. S., \& Hung, Y. P. (2011, June). Ordinal hyperplanes ranker with cost sensitivities for age estimation. In CVPR 2011 (pp. 585-592). IEEE.

[17] Wang, X., Guo, R., \& Kambhamettu, C. (2015, January). Deeplylearned feature for age estimation. In 2015 IEEE Winter Conference on Applications of Computer Vision (pp. 534-541). IEEE.

[18] Rothe, R., Timofte, R., \& Van Gool, L. (2016). Some like it hot-visual guidance for preference prediction. In Proceedings of the IEEE conference on computer vision and pattern recognition (pp. 5553-5561).

[19] Rothe, R., Timofte, R., \& Van Gool, L. (2018). Deep expectation of real and apparent age from a single image without facial landmarks. International Journal of Computer Vision, 126(2-4), 144-157.

[20] Guo, G., \& Mu, G. (2013, April). Joint estimation of age, gender and ethnicity: CCA vs. PLS. In 2013 10th IEEE International Conference and Workshops on Automatic Face and Gesture Recognition (FG) (pp. 1-6). IEEE.

[21] Guo, G., \& Mu, G. (2011, June). Simultaneous dimensionality reduction and human age estimation via kernel partial least squares regression. In CVPR 2011 (pp. 657-664). IEEE.

[22] Wan, J., Tan, Z., Lei, Z., Guo, G., \& Li, S. Z. (2018). Auxiliary demographic information assisted age estimation with cascaded structure. IEEE transactions on cybernetics, 48(9), 2531-2541. 